\title{
Review
}

\section{Cyanotic Congenital Heart Defects - literature review}

\author{
Vlad Drăgoi' ${ }^{1}$, Irina A. Horhoianu ${ }^{1,2}$, Răzvan V. Scăunașư ${ }^{2}$, Monica Cîrstoiuu, ${ }^{1,2}$ \\ ${ }^{1}$ Emergency University Bucharest Hospital, Department of Obstetrics and Gynecology, Bucharest, Romania \\ ${ }^{2}$ Carol Davila University, Faculty of General Medicine, Bucharest, Romania
}

\begin{abstract}
Congenital heart defects are one of the most interesting and important chapters regarding abnormal fetal growth pathology.

The objective of this article is to present a literature review for the main cyanotic congenital heart defects. The ones presented in the article are: tetralogy of Fallot, transposition of the great vessels, double outlet right ventricle, truncus arteriosus, total anomalous pulmonary venous circulation and additional information regarding very rare malformations such as pentalogy of Cantrell and Uhl anomaly.

An early and precise identification of congenital heart defects is an important step in an accurate follow-up of a potential problematic pregnancy. Knowing the sonographic aspect, associated pathology and the current available treatment procedures are vital for the fetal outcome and for the physician to adapt the right management in every situation that might appear during the pregnancy and in the neonatal period.
\end{abstract}

Keywords: congenital heart defects, tetralogy of Fallot, transposition of the great vessels, double outlet right ventricle, truncus arteriosus, total anomalous pulmonary venous circulation 


\section{Introduction}

When dealing with laparoscopy, similar to the open classical surgery, the approach difficulties in case of an acute cholecystitis are inevitable and require a good surgical laparoscopic training in order to diminish the intraoperative risk and to decide the most adequate therapeutic procedure. Fortunately, this type of pathology is a rear one in children and widely benefits from the advantages of laparoscopy.

Congenital heart defects are one of the most interesting and important chapters regarding abnormal fetal growth pathology. The objective of this article is to present a literature review for the main cyanotic congenital heart defects.

\section{Discussion}

\section{Tetralogy of Fallot (TOF)}

Tetralogy of Fallot is one of the most frequently diagnosed cyanotic congenital heart defects and is characterized by the presence of the following criteria: ventricular septal defect (VSD), overriding aorta, right ventricular outflow tract obstruction, right ventricular hypertrophy (usually diagnosed after birth). If an atrial septal defect (ASD) or a patent ductus arteriosus is present, then the malformation is called pentalogy of Fallot (1).

TOF can be accompanied by duodenal or esophageal atresia, diaphragmatic hernia (does not correlate with the usually high associated chromosomal risk), congenital lobar emphysema, tracheoesophageal fistula, VACTERL (vertebral anomalies - spina bifida, congenital scoliosis, hemivertebrae, anorectal anomalies - anal atresia, renal anomalies, poly/oligodactyly, tracheoesophageal fistula, cleft lip) $(2,3)$.

The main methods of diagnosis are echocardiography and MRI, used especially during pregnancy, alongside radiography and MDCT (multidetector CT) after birth. At the ultrasound examination the four chambers view does not present any particular significance. There can be identified: the presence of the VSD, different levels of pulmonary stenosis and the overriding aorta. Furthermore, one should also evaluate the pulmonary valve, the main pulmonary artery and arterial pulmonary branches because they could be hypoplastic.

In utero it is not possible to visualize the hypertrophy of the right ventricle and the pulmonary stenosis level is not significant. The most severe types of TOF are those associated with pulmonary atresia or with absent pulmonary valve syndrome (1).

Differential diagnosis is done with common arterial trunk, Ebstein's anomaly, pulmonary atresia, hypoplastic left heart syndrome, double outlet right ventricle (DORV) $(2,3)$.

TOF is rarely associated with $22 \mathrm{q} 11$ microdeletion (diagnosed using FISH technique $-15 \%$ of the cases with 22q11.2 or DiGeorge syndrome), however it has an equal distribution with trisomies 21 and 18 (3).

The treatment of TOF is surgical. A definitive corrective surgery is done at 3-6 months (other authors mention a 6-9 month period) from birth by closing the VSD using a patch and performing an infundibulectomy in order to remove the pulmonary artery outflow obstruction. In case of a major obstruction a palliative procedure such as placing a Blalock-Taussig (an aortopulmonary shunt between the left subclavian artery and the left pulmonary artery), Pott (an aortopulmonary shunt between the descendant thoracic artery and the left pulmonary artery) or Waterson (an aortopulmonary shunt between the ascending aorta and the right pulmonary artery) shunt should be done $(3,4)$.

In an article written by Tongsong et al. four fetuses with TOF were diagnosed (two cases at 25 weeks of gestation, one at 28 and another at 32). In the first fetus on the ultrasound examination the following elements were identified: normal size of the heart with an unmodified four chamber view, a subaortic VSD, an overriding aorta, a smaller main pulmonary trunk in comparison with the root of the aorta and a ratio 
regarding the size of the great vessels of 1.86 (the normal value reported was 0.90 ). The parents decided to terminate the pregnancy and the diagnosis was confirmed after the autopsy. The second case, diagnosed at the same age of 25 gestational weeks, had the following sonographic elements: the presence of a right ventricular hypertrophy on the four chamber view, a subaortic VSD, a small main pulmonary trunk and an aorta: pulmonary artery size ratio of 2.41 . The woman gave birth at 38 weeks to a $2740 \mathrm{~g}$ fetus and the diagnosis was confirmed following a neonatal echocardiography. There were some particular elements found in the case diagnosed at 28 weeks of gestation, such as: ascites, a globally enlarged heart, a mildly enlarged right ventricular hypertrophy - on the four chamber view, a subaortic VSD, an overriding aorta, a small main pulmonary trunk, a 1.31 ratio between the great vessels and a tricuspid and pulmonary regurgitation. In the last case presented by the authors the ultrasound examination showed: a normal sized heart, a VSD, agenesis of the pulmonary trunk, an overriding aorta. The patient delivered a $2010 \mathrm{~g}$ fetus at 33 weeks of gestation who survived after the surgical correction the cyanotic defect (5).

The main objective of the study done by Escribano et al. was to evaluate the importance and utility of mid second trimester fetal echocardiography in assessing the postnatal outcome of the cases diagnosed with TOF. They measured the morphological and functional cardiac parameters in two periods of time (between 1922 and 34-38 weeks of gestation) of 23 cases diagnosed with TOF. The study population was distributed into two groups: the EI group (newborns who needed a palliative procedure before the corrective surgery of the defect or the ones who needed a corrective surgery before 3 months of age) and the n-EI group (newborns who had a corrective procedure after 3 months of age). The second separation of the cases was done by type of corrective surgery that was performed: either a pulmonary valve sparing procedure (PVSS) or the placement of a patch (TAP). $96 \%$ of the patients survived and the main parameter used in order to evaluate the postnatal outcome was the pulmonary valve peak systolic velocity (PVPSV) measured between 19-22 weeks of gestation. A PVPSV of $\geq 87.5 \mathrm{~cm} / \mathrm{s}$ is associated with a very increased risk of TOF that requires a palliative surgery. Moreover, other parameters (which can be measured in the third trimester) that underline the accuracy of a correctly evaluated outcome are: the size of the pulmonary valve, the ratio of the pulmonary and aortic valves and the ratio between the great vessels (6).

Another paper of Bhat et al. had the purpose of comparing ultrasound examination and diagnosis of TOF at a pregnancy age of 12-17 weeks with those performed after the 17 weeks of gestation. The retrospective study was based on the hospital's experience between 2003 and 2008. A score based of the following criteria was created in order to assess the completeness of the sonographic examination: the pulmonary outflow obstruction and its severity, right ventricular outflow obstruction, pulmonary valve, pulmonary arteries including dimensions, pulmonary arterial flow, systemic and pulmonary venous anatomy, atrioventricular valves, ductus arteriosus, ductus flow, aortic arch morphology, sidedness and flow. The 35 cases diagnosed with TOF were divided into two groups: the 1st group - 10 cases (diagnosed at 12-17 weeks of gestation) and the 2nd group (diagnosed at > 17 weeks of gestation). The distribution of the 1st group was as following: seven fetuses with TOF with pulmonary stenosis and antegrade flow through the ductus arteriosus, one case of TOF/DORV and ectopia cordis, two of TOF alongside pulmonary atresia and bilateral superior vena cava, one of which was accompanied by left atrial isomerism. In the 2 nd group there were identified 
9 cases with pulmonary stenosis, 9 with pulmonary atresia, $3 \mathrm{TOF} / \mathrm{DORV}$ and 4 with absent pulmonary valve (7).

\section{Transposition of the great vessels (TGA)}

TGA is a cyanotic congenital heart defect characterized by an abnormal origin of the great vessels.

There are two types of TGA:

- L-loop transposition, congenitally corrected TGA, with both a atrioventricular and ventriculoarterial discordance: the left atrium is connected via a tricuspid valve to the right ventricle where the aorta emerges and the right atrium is connected via a mitral valve to the left ventricle where lies the origin of the pulmonary artery.

- D-loop transition, a pure ventriculo-arterial discordance (which is our main area of focus) (15). Differential diagnosis is done with corrected TGA and DORV (15).

Normally, TGA is not associated with other defects, the risk of non-chromosomal anomalies being very low. Some authors describe associations with maternal gestational diabetes, congenital anomalies of the coronary arteries, Ebstein's anomaly, tricuspid valve anomalies, septal defects and rarely with pulmonary stenosis $(2,3)$.

The diagnostic methods are identical to the ones presented at TOF. At the ultrasound examination we encounter a normal four chambers view. The diagnosis is made by evaluating the outflow tracts, where one could observe that the normal anatomical crossover between the two great vessels is absent. The aorta starts from the anterior part of the right ventricle and the pulmonary artery emerges from the posterior part of the left ventricle, therefore the normal three chambers view cannot be examined $(2,3)$.

The treatment is surgical: palliative or corrective. The palliative surgery is represented by the Senning and Mustard procedures performed at 3 months of age. The Rashkind surgery is used in TGA cases in which the ventricular sept is unaffected. The Jatene arterial switch is the main corrective procedure and implies the transection and positioning of the great vessels in their correct anatomical sites $(3,4)$. The Rastelli surgery is the standard procedure recommended in case of the association of a TGA, a DSV and a pulmonary stenosis (8).

A study carried out by Bertagna et al. had a population of 68 cases of TGA, which were divided into two groups: 32 of which were diagnosed prenatally $-47.1 \%$ (PND+) and 36 after birth (PND-). All of the 68 cases had a normal nuchal translucency measured between 11 and 13 weeks and 6 days of gestation. In the PND- group heart anatomy was considered to be normal in $75 \%$ of fetuses evaluated in the 2nd and 3rd trimesters. However, in the rest of the cases the fetal heart could not be correctly visualized in the 2 nd trimester, even though at the $3 \mathrm{rd}$ trimester scan it seemed to be normal. Therefore, the main inconvenient in evaluating and diagnosing correctly the pathology such as TGA is represented by maternal obesity. The authors suggested the fact that a reevaluation or using transvaginal ultrasound might increase the accuracy of an early diagnosis of TGA (9).

Escobar-Diaz et al. conducted a study, which had the main objective of evaluating the success rate of prenatal diagnosis of TGA (the form with an intact ventricular septum). The population studied was made up of 340 patients with TGA, 81 of which were diagnosed prenatally $(24 \%)$. The success rate of diagnosis went from $6 \%$ between 1992-1995 to $41 \%$ between the years 2008-2011. The newborns, which were prenatal diagnosed suffered a balloon atrial septostomy procedure (Rashkind surgery) earlier than those in which the defect was discovered after birth. An early diagnosis also meant that there were fewer cases where mechanic ventilation was needed (10).

\section{Truncus Arteriosus (TA)}

Truncus Arteriosus is a cyanotic congenital defect characterized by the presence of a VSD and a single artery, which emerges from the base of the heart, 
which has the role of ensuring systemic, coronary and pulmonary circulation. There are several ways of classifying this pathology. Based on the one made by Collett and Edwards there are 4 types of TA:

- Type 1 (the most frequent case) both the aorta and the main pulmonary artery have their origin in the truncus arteriosus

- Type 2- pulmonary arteries emerge from the posterior part of the truncus, one next to the other

- Type 3 (the rarest form) pulmonary arteries emerge from either of side of the truncus

- Type 4 is now considered to be a form of pulmonary atresia associated with a VSD, because none of the pulmonary artery branches emerge from the truncus (3).

The diagnostic methods are the same as the ones mentioned at TOF. At the ultrasound examination the four chamber view is usually normal. The diagnosis is made by identifying the VSD, the origin of the pulmonary arteries, the evaluation of the truncal valve which is frequently connected to both ventricles and can be associated with dysplasia, regurgitation or stenosis. Differential diagnosis is made with pulmonary atresia associated with VSD. There is a high-risk association with 22q11 microdeletion (20-30 $\%$ of cases) and a rare link with trisomies 13 and 18 was also reported. Regarding other defects, truncus arteriosus may be accompanied by an interrupted aortic arch, a right-sided aortic arch or by the CHARGE syndrome (coloboma, congenital heart defects, intellectual disability, genital hypoplasia, deafness) $(3,11)$.

The treatment is surgical and is performed in the neonatal period with the closure of the VSD, the repair of the truncal valve, the placement of an allograft between the right ventricle and the pulmonary arteries which are first transected from the truncus arteriosus and then reattached (2).

In an article written by Swanson et al. 136 patients diagnosed in utero or less than 60 days of age with TA between 1992-2007 were analyzed. $32 \%$ of the population was diagnosed prenatally; however 5 of these cases were false positive. Out of 28 fetuses diagnosed at less than 24 weeks of gestation, 19 did not survive (in 17 cases a termination of pregnancy was decided and 2 cases were due to intrauterine death). 19 patients diagnosed in utero with TA survived after birth, however two died before a corrective procedure was performed and four a short while after undergoing surgery (12).

\section{Double Outlet Right Ventricle (DORV)}

DORV is a cyanotic congenital malformation in which both the aorta and the pulmonary artery emerge from the right ventricle.

There are many types of DORV. Regarding the position of the great vessels, DORV can be classified as follows:

- Side by side

- Abnormal right side positioning

- Abnormal left side positioning $(3,4)$

Another way to classify DORV is by the type of VSD it is associated with:

- DORV with a subaortic VSD

- DORV with a subpulmonary VSD (Taussig-Bling heart)

- DORV with a VSD situated under the great vessels (doubly committed)

- DORV with a VSD, which is not situated in the proximity of the great vessels (non/ un committed) (3, $4)$.

The diagnostic methods are the same as the ones mentioned at TOF. At the ultrasound examination the four chambers view does not help in establishing the diagnosis. The great vessels outflow tracts are parallels, both ventricles are normal in size (if there is not an underlying atrioventricular valve atresia). The next step is to identify the VSD and its location (3).

Differential diagnosis is done with TOF and TGA. The risk of DORV being associated with chromosomal anomalies is relatively high (12-45\%), especially with trisomies 18 and 13 and in fewer cases with trisomy 21 and DiGeorge syndrome. That being said, karyotyping 
is a must. DORV can be accompanied by both cardiac defects such as: congenital pulmonary stenosis, coarctation of the aorta, right sided aortic arch, anomalous pulmonary venous circulation, as well as extracardiac ones, especially of gastrointestinal nature (tracheoesophageal fistula) or central nervous system malformations.

The treatment is surgical and is highly dependent on the type of DORV diagnosed and the severity of the defect. It has to be carried out in the neonatal period and the main objectives are: establishing an unobstructed flow at both outflow tracts and the closure of the ventricular septal defect. If DORV is associated with a subaortic VSD, the procedure used is similar to the one presented at TOF. If the VSD is a subpulmonary one, an arterial switch will be placed. Furthermore, in the presence of an obstruction at the pulmonary artery outflow tract a simple or modified (Rastelli) REV procedure (Réparation à l'Etage Ventriculaire) will be performed $(3,4)$.

Kim et al. conducted a study in which there were identified 19 cases with DORV out of a total of 987 ultrasound examinations performed. 3 out of 19 cases ended with the termination of the pregnancy and 4 out of the 13 cases confirmed with DORV survived 28 days after birth. Prenatal evaluation of the VSD type had a success rate of $100 \%$ (14 out of 14 cases) and had the following results: 8 VSD were subpulmonary, 6 subaortic, 2 uncommitted and 1 double committed. The same studies also suggest the fact that the outcome in case of pathology such as DORV is poor and associated with a mortality of $69 \%$ (13).

In another study which had the purpose of evaluating DORV cases diagnosed prenatally, Hartge et al. discovered 21 cases who underwent fetal karyotyping resulting in 3 fetuses with trisomy 18 and one with trisomy $21.61,19 \%$ of the fetuses were alive at birth, four of these suffering Rastelli maneuvers, one a Fontan intervention and three a resection of a coarctation of the aorta (14).
Gedikbasi et al. identified in a retrospective study 21 fetuses with DORV. 7 of these cases ended with the pregnancy being terminated of which 3 cases also had associated chromosomal anomalies - 1 case of trisomy 21 and 2 of trisomy 18 - and 1 was accompanied by the presence of an encephalocele. The success rate of the prenatal diagnosis (the VSD, the outflow obstruction of the great vessels and their spatial relationship) was of $87,5 \%$ (14/16 cases). The poor outcome of this congenital heart disease is further emphasized by this study in which only 2 newborns survived beyond 6 months of age (15).

Total Anomalous pulmonary venous circulation (TAPVC)

TAPVC is a cyanotic congenital heart defect in which pulmonary venous return is carried out abnormally at any heart level with the exception of the left atrium and all the four pulmonary veins establish aberrant connections.

After Darling's classification these are the following types of TAPVC:

- Type 1 - supracardiac (the most frequent case) the trajectory of the abnormal pulmonary veins ends at a supracardiac level where they unite and form a left vertical vein

- Type 2 - cardiac - the pulmonary veins carry out their drainage into the coronary sinus and right atrium

- $\quad$ Type 3 - infracardiac - the pulmonary veins unite behind the left atrium thus creating a common descending vertical vein

- $\quad$ Type 4 - mixed (the rarest case) - the pulmonary veins establish abnormal connections at different levels of the heart $(3,10)$.

The diagnostic methods are the same as the ones mentioned at TOF. At the ultrasound examination the first signs that show the presence of a TAPVC are represented by a moderate ventricular disproportion and an enlarged pulmonary artery in comparison to the aorta. Furthermore, other signs that suggest such a defect are: the impossibility of establishing a direct venous connection with the left atrium (the absence of 
venous return), the visualization of a vein with an ascending or descending trajectory, the detection of a venous confluence space behind the left atrium $(3,4)$.

Differential diagnosis is done with coarctation of the aorta. TAPVC is frequently associated with complex defects, which are included in the situs ambiguous spectrum $(2,3)$.

The treatment is surgical and has to be carried out in the first two months of age. The procedure consists in establishing a correct anatomical link between the pulmonary veins and the left atrium $(2,3)$.

Ganesan et al. conducted a retrospective study, which included 27 patients diagnosed prenatally with TAPVC. The following types of TAPVC were identified: type 1 - supracardiac - 18 cases, type 2 cardiac -1 case, and type 3 - infracardiac ( 7 cases). The ultrasound elements, which were present in the majority of examinations, were the absence of a visible connection between the pulmonary veins and the left atrium $(100 \%)$ and the presence of visible venous confluence discovered at the four chambers view. Moreover, pulmonary venous abnormalities have been identified in 25 out of 26 fetuses at the Doppler spectral scan (16).

\section{Special mentions}

- Pentalogy of Cantrell

Pentalogy of Cantrell is a rare syndrome characterized by the following malformations: omphalocele, ectopia cordis, diaphragmatic, pericardium and cardiac defects (VSD, ASD - atrial septal defect, TOF, TAPVC or left ventricle diverticulum) $(17,18)$.

The diagnostic methods are the same as the ones mentioned at TOF.

The survival rate associated with this pathology is very low, especially if all the elements associated with the syndrome are present $(3,17)$.

- Uhl Anomaly

Uhl anomaly or the "parchment heart" is a very rare cyanotic congenital heart disease characterized by the almost total absence of the right ventricle myocardium, but with normal functioning of the tricuspid valve.

At the ultrasound examination one can see an enlarged right ventricle, with a thin and hypokinetic wall. Furthermore, a moderate regurgitation of the tricuspid valve, a pericardial effusion and polyhydramnios can be also noted (19).

Differential diagnosis is done with Ebstein's anomaly and arrhythmogenic right ventricular dysplasia $(19,20)$.

\section{Conclusions}

An early and precise identification of congenital heart defects, both cyanotic and non-cyanotic, is an important step in an accurate follow-up of a potential problematic pregnancy. Knowing the sonographic aspect, associated pathology and the current available treatment procedures is vital for the fetal outcome and for the physician to adapt an adequate management in every situation that might appear during the pregnancy and in the neonatal period. Of outmost importance is also the fact that ultrasound examinations should be done by properly trained OB-GYN doctors who are specialized in this domain and in fetal morphology in order to establish an early and proper diagnosis.

\section{References:}

1. Coady AM, Bower S. Twinning's Textbook of Fetal Abnormalities, 3rd Edition. Elsevier 2015; 336-93. ISBN: 978-0-7020-4591-2

2. Creasy R.K., Resnik R, Besnik R., Iams J.D., Lockwood C.J., Moore T., Greene M.F., Creasy and Resnik's Maternal-Fetal Medicine: Principles and Practice, 7th Edition. Elsevier 2014; 852-77. ISBN: 978-1-4557-1137-6

3. Paladini D, Volpe P. Ultrasound of Congenital Fetal Anomalies: Differential Diagnosis and 
Prognostic Indicators, Second Edition. CRC Press 2014; 312-515. ISBN: 9781466598966, https://doi.org/10.1201/b16779

4. Kline-Fath B, Bahado-Singh R, Bulas D, Fundamental and Advanced Fetal Imaging Ultrasound and MRI, Wolters Kluver 2015; 54073. ISBN: 9781451175837

5. Tongsong $\mathrm{T}$, Sittiwangkul $\mathrm{R}$, Chanprapaph $\mathrm{P}$, Sirichotiyakul S. Prenatal Sonographic Diagnosis of Tetralogy of Fallot. J Clin Ultrasound. 2005; 33(8): 427-31. PMID: 16240425, https://doi.org/10.1002/jcu.20147

6. Escribano D, Herraiz I, Granados M, Arbues J, Mendoza A, Galindo A. Tetralogy of Fallot: prediction of outcome in the mid-second trimester of pregnancy. Prenat Diagn. 2011; 31(12): $\quad$ 1126-33. $\quad$ PMID: 21928295 , https://doi.org/10.1002/pd.2844

7. Bhat AH, Kehl DW, Tacy TA, Moon-Grady AJ, Hornberger LK. Diagnosis of Tetralogy of Fallot and Its Variants in the Late First and Early Second Trimester: Details of Initial Assessment and Comparison with Later Fetal Diagnosis. Echocardiography. $\quad 2013 ; \quad 30(1)$ : $\quad 81-7$. PMID: 22963380, https://doi.org/10.1111/j.15408175.2012.01798.X

8. Squarcia U, Macchi C. Transposition of the great arteries. Curr Opin Pediatr. 2011; 23(5): 518-22. PMID: 21849902, https://doi.org/10.1097/MOP.0b013e32834aa551

9. Bertagna F, Rakza T, Vaksmann G, RamdaneSebbane N, Devisme L, Storme L, Francart C, Vaast P, Houfflin-Debarge V. Transposition of the great arteries: factors influencing prenatal diagnosis. Prenat Diagn. 2014; 34(6): 534-7. PMID: 24532355, https://doi.org/10.1002/pd.4343

10. Demos TC, Posniak HV, Pierce KL, Olson MC, Muscato M. Venous anomalies of the thorax. AJR
Am J Roentgenol. 2004; 182(5): 1139-50. PMID: 15100109, https://doi.org/10.2214/ajr.182.5.1821139

11. Perloff JK, Marelli A. Perloff's Clinical Recognition of Congenital Heart Disease, 6th Edition. Elsevier 2012; 414-576. ISBN: 978-14377-1618-4

12. Swanson TM, Selamet Tierney ES, Tworetzky W, Pigula F, McElhinney DB. Truncus Arteriosus: Diagnostic Accuracy, Outcomes, and Impact of Prenatal Diagnosis. Pediatr Cardiol. 2009; 30(3): 256-61. PMID: 19015910, https://doi.org/10.1007/s00246-008-9328-7

13. Kim N, Friedberg MK, Silverman NH. Diagnosis and prognosis of fetuses with double outlet right ventricle. Prenat Diagn. 2006; 26(8): 740-5. PMID: 16807954, https://doi.org/10.1002/pd.1500 14. Hartge DR, Niemeyer L, Axt-Fliedner R, Krapp M, Gembruch U, Germer U, Weichert J. Prenatal detection and postnatal management of double outlet right ventricle (DORV) in 21 singleton pregnancies. J Matern Fetal Neonatal Med. 2012; 25(1): 58-63. PMID: 21410422, https://doi.org/10.3109/14767058.2011.561387

15. Gedikbasi A, Oztarhan K, Gul A, Sargin A, Ceylan Y. Diagnosis and Prognosis in DoubleOutlet Right Ventricle. Am $J$ Perinatol. 2008; 25(7): 427-34. PMID: 18720325, https://doi.org/10.1055/s-0028-1083840

16. Ganesan S, Brook MM, Silverman NH, MoonGrady AJ. Prenatal findings in total anomalous pulmonary venous return: a diagnostic road map starts with obstetric screening views. J Ultrasound Med. 2014; 33(7): 1193-207. PMID: 24958406, https://doi.org/10.7863/ultra.33.7.1193

17. Kheir AEM, Bakhiet EA, Elhag SMM, Karrar MZ. Pentalogy of Cantrell: case report and review of the literature. Sudan J Paediatr. 2014; 14(1): 85-88. PMCID: PMC4949922 
18. Restrepo SM, Cerqua A, Turek WJ. Pentalogy of Cantrell with ectopia cordis totalis, total anomalous pulmonary venous connection, and tetralogy of Fallot: a case report and review of the literature. Congenit Heart Dis. 2014; 9(4): $\quad$ E129-34. PMID: 23750667, https://doi.org/10.1111/chd.12101

19. Cardaropoli D, Russo MG, Paladini D, Pisacane C, Caputo S, Giliberti P, Calabrò R. Prenatal echocardiography in a case of Uhl's anomaly.
Ultrasound Obstet Gynecol. 2006; 27(6): 713-4. PMID: 16710881, https://doi.org/10.1002/uog.2798

20. Escobar-Diaz MC, Freud LR, Bueno A, Brown DW, Friedman KG, Schidlow D, Emani S, Del Nido PJ, Tworetzky W. Prenatal diagnosis of transposition of the great arteries over a 20 -year period: improved but imperfect. Ultrasound Obstet Gynecol. 2015; 45(6): 678-82. PMID: 25484180, https://doi.org/10.1002/uog.14751 